\title{
LITERATUR
}

\section{Die Anfangsjahre der Europäischen Kommission aus Sicht der historischen Integrationsforschung}

\author{
Michael Gehler*
}

Die Kommissionen der Europäischen Wirtschaftsgemeinschaft (EWG) und Europäischen Gemeinschaften (EG) setzten sich aus Funktionseliten zusammen, die den Integrationsprozess wesentlich mitgestalteten und durch administrative sowie konzeptionelle Arbeit zum Erfolg der europäischen Integration ganz erheblich beitrugen. In der hier vorgestellten Publikation über die Anfangsjahre der Europäischen Kommission wird der Zeitraum 1958-1972 behandelt. Es handelt sich um ein Sammelwerk von Einzelbeiträgen und zugleich um eine Quellendokumentation und Chronologie, die zudem mit Organigrammen, Personenregister und Tabellen ausgestattet ist. Darin werden die verschiedensten Facetten dieses supranationalen Herzstücks der Europäischen Gemeinschaften aufgezeigt.

\section{Prägende Personen}

Ein erster großer Teil dieser Publikation widmet sich intensiv dem Aufbau, der Organisation, der Struktur und den Individuen, die diese Institution getragen und mit Leben erfüllt haben. Unter Federführung des ausgewiesenen Integrationshistorikers Michel $\mathrm{Du}$ moulin werden die Arbeiten des sogenannten Interimsausschusses dargestellt, der nach der Unterzeichnung der Römischen Verträge am 25. März 1957 vom April 1957 bis zum Inkrafttreten des „Gemeinsamen Marktes“ (EWG) und von EURATOM am 1. Januar 1958 agierte. Wilfried Loth und Marie-Thérèse Bitsch widmen sich der Kommission unter ihrem Präsidenten Walter Hallstein, der von 1958 bis 1967 amtierte, Loth dem Wirken des ersten Kommissionspräsidenten in der
Europäische Kommission: Die Europäische Kommission 1958-1972 - Geschichte und Erinnerungen einer Institution, unter der Leitung von Michel Dumoulin, in Zusammenarbeit mit Marie-Thérèse Bitsch/Gérard Bossuat/Éric Bussière/ Julie Cailleau/Yves Conrad/Anaïs Legendre/ Matthieu Lethé/Wilfried Loth/Jan van der Harst/ Arthe Van Laer und Antonio Varsori, auf Initiative der Europäischen Kommission unter Mitwirkung ehemaliger europäischer Beamter, Amt für amtliche Veröffentlichungen der Europäischen Gemeinschaften: Luxemburg 2007, ISBN 97892-79-05493-8, 669 Seiten, 30,- €. Verfügbar in deutscher, englischer, französischer, italienischer und niederländischer Sprache.

Geschichte der europäischen Integration als einem „überzeugten Europäer“. Außerdem beschäftigt sich Loth mit der von de Gaulle provozierten Krise des ,leeren Stuhles“ (,chaise vide'), die für ein gutes halbes Jahr (1965/66) zu einer Totalblockade des EWGMinisterrates führte und erst durch den sogenannten „Luxemburger Kompromiss“ (Januar 1966) überwunden werden konnte. Doch damit hatte die Kommission Prestige eingebüßt. Yves Conrad widmet sich dem Nachfolger Hallsteins, dem Belgier Jean Rey, der ebenfalls ,überzeugter Europäer“, aber im Unterschied zu Hallstein ein ,zurückhaltender Optimist" war. Bitsch beleuchtet die ersten Jahre der gemeinsamen Kommission (1967-1972) nach der Fusion der Organe von EGKS, EWG, EURATOM. Der an der Universität Padua lehrende italienische Integrationsspezialist Antonio Varsori beschäftigt sich mit der verkürzten Präsidentschaft von Franco Maria Malfatti, während sich der niederländische

\footnotetext{
* Prof. Dr. Michael Gehler, Geschäftsführender Leiter, Institut für Geschichte, Stiftung Universität Hildesheim.
} 
Historiker Jan van der Harst dem Agrarpolitiker Sicco Mansholt und dessen Landwirtschafts-Reformplänen zuwendet. Das Kollegium der Kommissare als ,neuer Beamtentyp ist Anliegen von Bitsch und Conrad. Der französische Kommissar Émile Noël ist Untersuchungsgegenstand von Gérard Bossuat. Mit dem Verwaltungsaufbau und der Arbeitsweise der Kommission befasst sich Dumoulin. Dumoulin und Conrad nehmen die EGBeamten in Brüssel zum Untersuchungsgegenstand. Die Frage des Sitzes der Kommission ist ebenfalls ein Thema für Dumoulin und Matthieu Lethé.

\section{Politikfelder}

Ein zweiter großer Teil dieser breit angelegten Veröffentlichung umfasst die wesentlichen Politikfelder der EWG-/EG-Kommission. Den Weg von der sektoriellen Integration der Montanunion zum „Gemeinsamen Markt“ und zur Wettbewerbspolitik schildert Éric Bussière, die "Gemeinsame Agrarpolitik" als „vorrangiges Aktionsfeld“ behandelt van der Harst und die Rolle der Kommission in den auswärtigen Beziehungen analysieren Bossuat und Anaïs Legendre. Den für Frankreich relevanten Übergang der Politik der Assoziierung der ,überseeischen Länder und Hoheitsgebiete" zur Entwicklungshilfe behandelt Dumoulin und Bussière verfolgt die „Versuche einer Wirtschafts- und Währungspolitik“. Varsori beleuchtet sowohl die Regionalpolitik als „Anfänge einer Solidarität“ der Europäischen Gemeinschaften als auch die Ursprünge der europäischen Sozialpolitik. Die Verkehrspolitik als „Bastion nationaler Pfründe" betrachtet Dumoulin in einem weiteren Beitrag, während sich Bussière den Problemen der Anfänge einer europäischen Industriepolitik widmet. Das Feld der Energie als Politik von „Synergien durch Fusion“ im Rahmen von EURATOM ist Thema des Artikels von Julie Cailleau. Bussière und Arthe
Van Laer behandeln Forschung und Technologie und benennen dabei die ,sechsfache Vormundschaft" der EWG-Mitgliedstaaten über die „,ewig minderjährige Kommission“. Die Entwicklung der Informationspolitik untersucht Dumoulin und die Erweiterung und Findung neuer Rollen der Kommission van der Harst.

\section{Grundlagenwerk}

Eine Zeittafel, Organisationspläne der Kommission aus den Jahren 1964, 1968 und 1972 sowie ein Quellenverzeichnis und ein ausführliches nach Publikationsarten gegliedertes Literaturverzeichnis, Autorennachweise, Personenregister sowie eine Tabelle mit Kürzeln und Abkürzungen runden eine eindrucksvolle Publikation ab, die zwar von der Kommission initiiert und von der Europäischen Union herausgebracht wurde, aber in jeder Hinsicht streng wissenschaftlichen Ansprüchen gerecht wird und ein unverzichtbares Grundlagen- und Standardwerk für die weitere historische Integrationsforschung darstellt.

\section{Spezifische Identifikation}

Die ersten Beamten der Kommission waren im Sinne einer Aufbauarbeit regelrechte Pioniere und dieser neuen Institution in wachsender Treue verbunden, während die Kommissare von den Regierungen der Mitgliedstaaten für eine oder mehrere Amtszeiten nach Brüssel entsandt wurden. Die Arbeitserfahrungen führten zu einer spezifischen Identifikation der Beamten mit der Kommission. Vorbilder der europäischen Integrationspolitik hatten dabei einen erheblichen Einfluss auf die Kommissare und Spitzenbeamten. Wie Katja Seidel $^{1}$ in ihren jüngsten Forschungen nachgewiesen hat, war der zuständige Vizepräsident der Kommission, Sicco Mansholt, im Kontext der Konzeptionen der europäischen Landwirtschaftspolitik eine Vorbild gebende Persönlichkeit. Er ließ ein spezifisches Rekru-

1 Katja Seidel: „So ein Mix von Menschen“. Gruppenbiographische Studien zu Beamten in der EWG-Kommission, in: Michael Gehler unter Mitarbeit von Andreas Pudlat (Hrsg): Vom Gemeinsamen Markt zur Europäischen Unionsbildung. 50 Jahre Römische Verträge 1957-2007. From Common Market to European Union Building. 50 years of the Rome Treaties 1957-2007, Wien/Köln/Weimar im Erscheinen. 
tierungsmilieu entstehen und förderte ein positives Arbeitsklima. Nicht unwesentlich waren Vorarbeiten im Sekretariat des Ministerrats und der Hohen Behörde der EGKS in Luxemburg aber auch jene durch die OEEC in Paris. Diese Institutionen und Organisationen als Rekrutierungsfeld von inhaltlicher Expertise und personeller Erfahrung haben in dem hier besprochenen Werk etwas zu wenig Berücksichtigung gefunden. Die Verhandlungen zur internationalen Landwirtschaftspolitik im sogenannten, green pool ${ }^{\text {* }}$ oder im Rahmen der Handels- und Zahlungsverkehrsliberalisierung haben im Rahmen der OEEC schon Anfang der 1950er Jahre eine Art Kontaktbörse gebildet. Seidel hat außerdem ermittelt, dass nur wenige Beamte vor Eintritt in die Kommission der Europabewegung angehört hatten oder in europäischen Studentenorganisationen engagiert waren. Neben Mansholt waren es Führungsleute wie die Deutschen Walter Hallstein oder Hans von der Groeben, die zu Leitfiguren für viele Kommissionsbeamte avancierten. Von der Groebens Arbeitsmethoden ähnelten in vielem jenen von Mansholt. Durch ein Klima der Offenheit wurden Probleme sowohl gemeinsam diskutiert als auch angegangen und damit Teamgeist und Teamarbeit möglich. Das hohe Arbeitspensum und die gemeinsamen beruflichen Erfolgserlebnisse förderten die Identifikation mit den Zielvorstellungen und Werten dieser supranationalen Institution. Für viele Kommissionsmitglieder entstand der Eindruck einer ,Erschaffungsphase', so Seidel, das heißt das belebende Gefühl, an einem neuen Werk beteiligt zu sein.

\section{Kollektivbiographische Untersuchungen}

Im weiteren Verlauf verlor jedoch die Kommission Elan und Impetus und wurde mehr und mehr zu einer Art Verwaltung, zumal unter sechs Staaten noch ein stärkerer Gemeinschaftsgeist vorhanden gewesen war als später unter den neun oder zwölf Vertretern der Mitgliedstaaten. Die kollektivbiographischen Untersuchungen von Seidel ergänzen die hier zu besprechende ausgezeichnete Publikation und zeigen, dass ein Großteil der Beamten bereits in nationalen Verwaltungen vorgeprägt und sozialisiert worden war. Sogenannte, Beamte auf Zeit', die nur für ein paar Jahre in die Kommission abgesandt wurden und dann wieder in die nationalen Verwaltungen zurückgingen, gehören auch zur Sozial- und Identifikationsstruktur der EWG-Kommission. Entscheidend bleibt, dass die gemeinsame generationelle Erfahrung, die Identifikation mit dem Politikbereich gepaart mit teilhabenden Arbeitsmethoden und der Präsenz von Vorbildern eine starke Identifikation der Kommissionsbeamten ermöglicht haben. Sie sind als Faktoren der Europäisierung zu bewerten, die einerseits zur Homogenisierung andererseits aber auch zu einer Desensibilisierung der Beamten für nationale Probleme beitrugen. Seidel gelangt sogar zu der Gesamtdeutung, dass Beamte der sogenannten ,45er-Generation" aus einer transnationalen Kriegsgenerationen-Identität heraus miteinander verbunden waren und dadurch ihre Europäisierung erfuhren. In den ersten Jahren von 1958 bis Ende der 1960er Jahre war die Kommission eine relativ kleine Verwaltung, in der persönliche Beziehungen unter den Beamten und zwischen den Beamten und dem Kommissar einfacher $\mathrm{zu}$ entwickeln waren. Durch die Zunahme der Aufgabenbereiche wurde es unvermeidlich, dass die Verwaltung anwuchs und persönliche Beziehungen schwerer aufrecht zu erhalten waren.

Auf weitere ergänzende Forschungen zu den Kommissionen der 1970er, 1980er und 1990er Jahre darf man gespannt sein. Mit diesem hier besprochenen fundamentalen wie mustergültigen Werk ist der Geschichtsforschung jedenfalls ein großer Schritt bis in die Anfänge der 1970er Jahre gelungen. 